\title{
Plasma FIB Spin Milling for 3D Residual Stress Measurements
}

B. Winiarski ${ }^{1,2 *}$, C. Rue $^{3}$ and P.J. Withers ${ }^{2}$

1. Thermo Fisher Scientific, V.Pecha 12, Brno 627 00, Czech Republic

2. The Henry Royce Institute, School of Materials, The University of Manchester, M13 9PL, U.K.

3. Thermo Fisher Scientific, 5350 NE Dawson Creek Dr, Hillsboro, OR 97124, USA

*Corresponding author: Bartlomiej.Winiarski@ thermofisher.com

Residual stresses arise in most materials as a consequence of processing and/or in-service loading. Depending on their sign, magnitude, spatial distribution, and the scale over which they equilibrate, residual stresses can alter the mechanical and functional performance. Consequently, their quantification is of great importance across many sectors. Whilst there exists a plethora of techniques for measuring stress at the macroscale, few techniques are available at the micron scale [1-4], either laterally or with depth, especially for amorphous materials.

Measuring residual stress at the sub-micron scale using focused ion beam - scanning electron microscope - digital image correlation (FIB-SEM-DIC) micro-hole drilling method imposes experimental challenges, because SEM images are subject to fluctuations that can introduce large artefacts, i.e. shift, stretch and shear, when using DIC. Recently a full-field, multi-axial computation technique was described for determining residual stresses using the hole-drilling method with DIC [5]. The computational method exploited the large quantity of data available from full-field images to ameliorate the effect of modest deformation sensitivity of DIC measurements. It also provided uniform residual stress sensitivity in all in-plane directions and accounts for artefacts that commonly occur within experimental measurements.

The advent of dual beam plasma focused ion beam - scanning electron microscope (PFIB-SEM) has made it possible to make very large, $>(100 \mu \mathrm{m})^{2}$ and smooth cross-sections for high resolution and large volume serial sectioning tomography [6]. Recently the micro-hole drilling method was combined with PFIB-SEM automated serial sectioning allowing measuring residual stresses in 3D dimensions with high spatial resolution over large volumes [7]. Plasma DualBeam microscopes equipped with negative tilt stages are routinely used for delayering of electronic components by PFIB spin milling (PFIB-SM) at glancing angles on surfaces. PFIB-SM is somewhat similar to broad ion beam serial sectioning (recently developed in [8]) but allows superior control of the delayering process.

In this work we combine PFIB spin mill delayering using Thermo Scientific ${ }^{\mathrm{TM}}$ multi-ion Hydra $^{\mathrm{TM}}$ PFIBSEM and PFIB-SEM-DIC micro-hole residual stress measurements with SEM artifacts correction. This has allowed us to map volumetric distribution of in-plane residual stresses and with a micron scale resolution. We achieved repeatable removal of layers dozens of $\mathrm{nm}$ thick, as measured by the crossholes method proposed in [8]. Here we present an analysis of this approach applied to laser shot peened bulk metallic glass specimen [9]. The high lateral RS gradients (on the order of dozens $\mathrm{MPa} / \mu \mathrm{m}$ ) make many far-field experimental techniques unsuitable. Our approach overcomes these difficulties. We show the $3 \mathrm{D}$ residual stress distribution over $500 \times 500 \mu \mathrm{m}^{2}$ area and depths of a few microns from the shot peened surface. 
References:

[1] B Winiarski and PJ Withers., Experimental Mechanics 52/4 (2012), p. 417-428.

[2] B Winiarski at al., Metallurgical and Materials Transactions A41/7 (2010), p. 1743-1751.

[3] S Massl et al., Scripta Materialia 59/5 (2008), p. 503-506.

[4] AM Korsunsky at al., Surface Coating Technology 205 (2010), p. 2393-2403.

[5] GS Schajer, B Winiarski and PJ Withers. Experimental Mechanics 53 (2013), p. 255-265.

[6] TL Burnett, R Kelley, B Winiarski et al., Ultramicroscopy 161 (2016), p. 119-129.

[7] B Winiarski at al., 3D MS Conference (2016), p. 34.

[8] B Winiarski at al., Ultramicroscopy 172 (2017), p. 52-64.

[9] Y Cao at al., Scientific Reports 5 (2015), p. 10789.
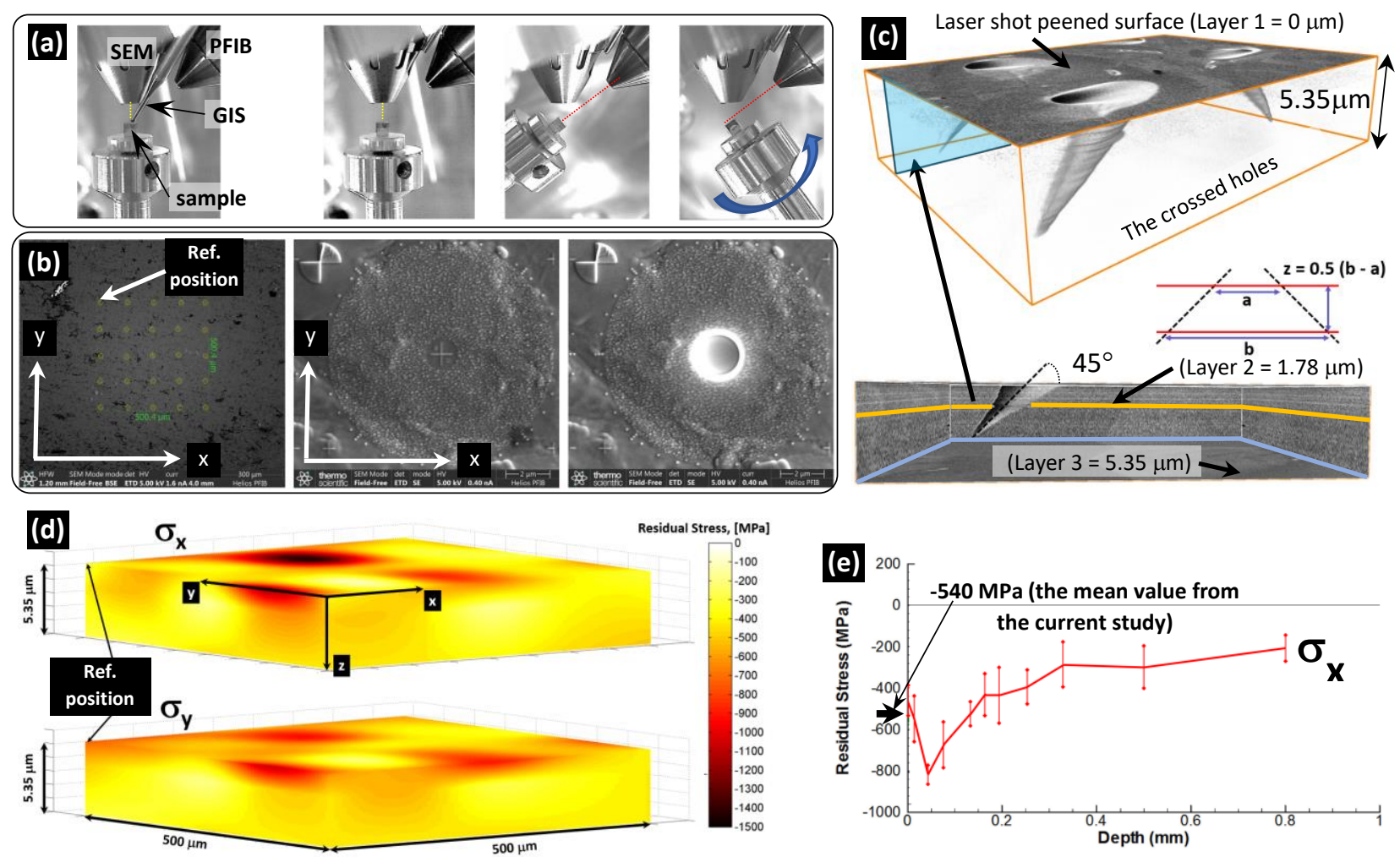

Figure 1. Shows the experimental setup and results. (a) IR photographs show geometric configurations of the 3D residual stress measurements using PFIB spin milling. Left to right: SEM-assisted nano-Pt markers deposition; SEM imaging; PFIB micro-hole drilling; PFIB glancing angle layer removal $(12 \times$ $30^{\circ}$ angular positions). (b) Residual stress measurements. Left to right: SEM images show spacing (125 $\mu \mathrm{m})$ of micro-holes array $(5 \times 5)$ on the laser shot peened sample surface; nano-Pt dot markers for Digital Image Correlation; a micro hole, $2 \mu \mathrm{m}$ diameter. (c) The crossed holes method for measuring the thickness of removed material. (d) The 3D residual stress distribution in the superficial surface layers. (e) Residual stress depth profile measured using the micro-slitting method in [9]. 